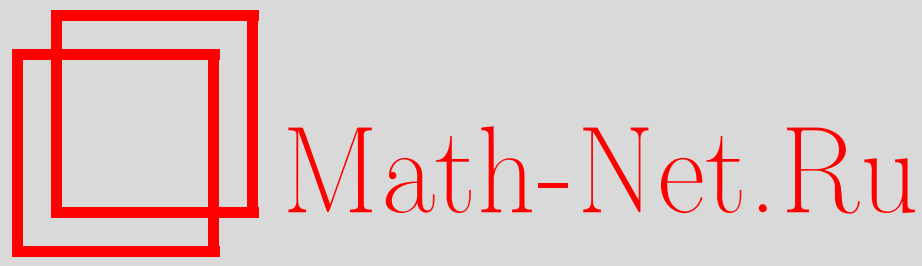

Т. В. Дудникова, Вывод гидродинамических уравнений для решетчатых систем, ТМФ, 2011, том 169, номер 3, 352-367

DOI: https://doi.org/10.4213/tmf6735

Использование Общероссийского математического портала Math-Net.Ru подразумевает, что вы прочитали и согласны с пользовательским соглашением http://www.mathnet.ru/rus/agreement

Параметры загрузки:

IP : 54.80 .73 .141

26 апреля 2023 г., 13:17:29

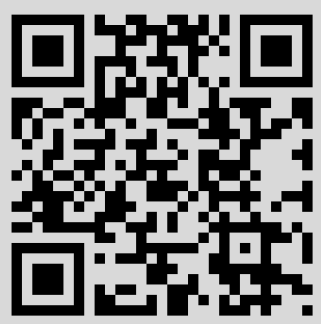




\title{
ФИЗИКА
}

Том 169, № 3

декабрь, 2011

2011 г.

Т. В. Дудникова*

\section{ВЫВОД ГИДРОДИНАМИЧЕСКИХ УРАВНЕНИЙ ДЛЯ РЕШЕТЧАТЫХ СИСТЕМ}

\begin{abstract}
Изучается динамика решетчатых систем в $\mathbb{Z}^{d}, d \geqslant 1$. Предполагается, что начальные данные - случайные функции. Вводится семейство начальных мер $\left\{\mu_{0}^{\varepsilon}, \varepsilon>0\right\}$. Предполагается, что меры $\mu_{0}^{\varepsilon}$ являются локально однородными или "мало меняются" при пространственных сдвигах порядка $o\left(\varepsilon^{-1}\right)$ и неоднородными при сдвигах порядка $\varepsilon^{-1}$; кроме того, корреляции мер $\mu_{0}^{\varepsilon}$ убывают равномерно по $\varepsilon$ на больших расстояниях. Для любых $\tau \in \mathbb{R} \backslash 0, r \in \mathbb{R}^{d}$ и $\kappa>0$ рассматриваются распределения случайного решения в моменты времени $t=\tau / \varepsilon^{\kappa}$ в точках, близких $\mathrm{k}[r / \varepsilon] \in \mathbb{Z}^{d}$. Основная цель - изучение асимптотики этих распределений при $\varepsilon \rightarrow 0$ и вывод предельных гидродинамических уравнений типа Эйлера и Навье-Стокса.
\end{abstract}

Ключевые слова: гармонические кристаллы, задача Коши, случайные начальные данные, слабая сходимость мер, гауссовские меры, гидродинамический предел, уравнение Эйлера, уравнение Навье-Стокса.

\section{1. ВВЕДЕНИЕ}

Настоящая статья посвящена математическим проблемам обоснования статистической физики и продолжает работы [1], [2], в которых приведен вывод гидродинамических уравнений из гамильтоновых уравнений движения системы частиц. Один из подходов к решению этой задачи заключается в изучении гидродинамического поведения различных упрощенных моделей взаимодействия частиц, например одномерных твердых стержней [3], одномерных осцилляторов на решетке [4]-[6]. Отметим также большое количество работ, посвященных моделям стохастической динамики (см., например, работы [7]-[9] и ссылки в них). Подробное обсуждение результатов можно найти в обзорных работах [10]-[13].

В настоящей работе в качестве модели рассматриваются гармонические кристаллы в $\mathbb{Z}^{d}, d \geqslant 1$. В гармоническом приближении кристалл характеризуется отклонениями $u(z, t) \in \mathbb{R}^{n}, n \geqslant 1, z \in \mathbb{Z}^{d}$, атомов кристалла от их положения равновесия. Поле $u(z, t)$ удовлетворяет дискретному волновому уравнению. Гармонические кристаллы можно рассматривать как обобщение на многомерный случай модели одномерных осцилляторов на решетке (см. работы [4]-[6], [14]).

*Электростальский политехнический институт, Электросталь, Московская обл., Россия. E-mail: tdudnikov@mail.ru 
Вывод гидродинамических уравнений связан с проблемой сходимости к равновесной мере. Для рассматриваемой модели такая сходимость была доказана в работах [15], [16]. Кратко сформулируем данный результат. Предполагается, что начальные данные - это случайная функция с распределением $\mu_{0}$. На меру $\mu_{0}$ налагаются следующие условия: $\mu_{0}$ обладает нулевым средним, имеет конечную среднюю плотность энергии и удовлетворяет условию перемешивания. Мы изучаем распределение $\mu_{t}$ случайного решения $u(\cdot, t)$ в моменты времени $t \in \mathbb{R}$. В работе [15] доказано, что меры $\mu_{t}$ при $t \rightarrow \infty$ слабо сходятся к некоторой предельной мере, которая является гауссовской. Для одномерной цепочки гармонических осцилляторов этот результат был доказан в работе [14], сходимость к равновесному распределению была доказана также для систем, описываемых уравнениями в частных производных [17], [18], и для кристалла, взаимодействующего со скалярным волновым полем [19].

Для вывода гидродинамических уравнений применяется специальная так называемая гидродинамическая предельная процедура. Она заключается в следующем. Вводится малый параметр $\varepsilon>0$, задающий отношение между масштабами измерений в "макро-" и "микроскопических" переменных пространства и времени, и рассматривается семейство начальных мер $\left\{\mu_{0}^{\varepsilon}, \varepsilon>0\right\}$, удовлетворяющих некоторым условиям (см. условия V1 и V2 в разделе 1.2). В частности, предполагается, что, во-первых, меры $\mu_{0}^{\varepsilon}$ являются локально пространственно-однородными или "мало меняются" при сдвигах порядка меньше $\varepsilon^{-1}$ и неоднородными при сдвигах порядка $\varepsilon^{-1}$; во-вторых, корреляции мер $\mu_{0}^{\varepsilon}$ убывают равномерно по $\varepsilon$ на больших расстояниях.

Для вывода уравнений типа Эйлера в работе [1] используется так называемое гиперболическое изменение масштаба, т. е. $t=\tau / \varepsilon$ и $z=[r / \varepsilon]$, где через $t, z$ обозначаются микроскопические переменные, а через $\tau, r$ - макроскопические переменные времени и пространства ([·] - целая часть числа). Для любых $\tau \in \mathbb{R} \backslash 0$ и $r \in \mathbb{R}^{d}$ мы изучаем распределение $\mu_{\tau / \varepsilon, r / \varepsilon}^{\varepsilon}$ случайного решения $u(z, t)$ в точках пространства, близких к точке $[r / \varepsilon]$, и в моменты времени $\tau / \varepsilon$. Доказано (см. теорему 3.3 в [1]), что

$$
\lim _{\varepsilon \rightarrow 0} \mu_{\tau / \varepsilon, r / \varepsilon}^{\varepsilon}=\mu_{\tau, r}
$$

где $\mu_{\tau, r}$ - некоторая гауссовская мера. В частности, в работе [1] были выведены точные формулы для корреляционной матрицы $q_{\tau, r}\left(z-z^{\prime}\right)$ предельной меры $\mu_{\tau, r}$, из которых вытекает, что полученная в результате преобразования Фурье матричнозначная функция $\widehat{q}_{\tau, r}(\theta), \theta \in \mathbb{T}^{d}$, где $\mathbb{T}^{d}-d$-мерный тор $\mathbb{R}^{d} /(2 \pi \mathbb{Z})^{d}$, является решением следующего уравнения:

$$
\partial_{\tau} f(\tau, r, \theta)=i C(\theta) \nabla \Omega(\theta) \cdot \nabla_{r} f(\tau, r, \theta), \quad r \in \mathbb{R}^{d}, \quad \tau>0,
$$

где $C(\theta)=\left(\begin{array}{cc}0 & \Omega^{-1}(\theta) \\ -\Omega(\theta) & 0\end{array}\right)$ и $\Omega(\theta)$, грубо говоря, является дисперсионным соотношением кристалла (более детально результаты (1.1) и (1.2) сформулированы в п. 2.1 настоящей работы). Здесь и ниже через $\partial_{\tau}$ обозначается частная производная по $\tau$, через $\nabla_{r} f$ - градиент функции $f$ по переменной $r \in \mathbb{R}^{d}$, а точкой обозначено скалярное произведение в $\mathbb{R}^{d}$. Уравнение (1.2) можно рассматривать как аналог уравнения Эйлера для нашей модели. Аналогичные уравнения были выведены в работе [4] в случае $d=n=1$. Эти результаты были обобщены в статьях [1] и [2] на случай 
гармонических кристаллов во всем пространстве $\mathbb{Z}^{d}, d \geqslant 1$, и в полупространстве $\mathbb{Z}_{+}^{d}=\left\{z \in \mathbb{Z}^{d}: z_{1}>0\right\}$ соответственно.

Основной результат настоящей работы - это вывод уравнения для "следующего приближения" к уравнению Эйлера (1.2). Чтобы получить дополнительный член порядка $\varepsilon$ в (1.2), мы изучаем поведение распределений решения $u(z, t)$ в моменты времени порядка $\tau / \varepsilon^{2}$ и после соответствующей замены переменных выводим уравнение типа Навье-Стокса (точную формулировку результата см. ниже в теореме 3 и в следствии 2). В случае $d=n=1$ эти результаты были получены в работе [5]. Поэтому при доказательстве (см. раздел 3) будет использован как подход работы [5], так и методы работ [1], [15], разработанные для многомерных гармонических кристаллов. В приложении приводятся “поправки” более высокого порядка (порядка $\varepsilon^{k}$, $k \geqslant 2)$ к уравнению (1.2).

1.1. Модель. Мы изучаем динамику гармонических кристаллов в $\mathbb{Z}^{d}, d \geqslant 1$ :

$$
\begin{aligned}
& \ddot{u}(z, t)=-\sum_{z^{\prime} \in \mathbb{Z}^{d}} V\left(z-z^{\prime}\right) u\left(z^{\prime}, t\right), \quad z \in \mathbb{Z}^{d}, \quad t \in \mathbb{R}, \\
& u(z, 0)=u_{0}(z), \quad \dot{u}(z, 0)=u_{1}(z), \quad z \in \mathbb{Z}^{d} .
\end{aligned}
$$

Здесь $u(z, t)=\left(u_{1}(z, t), \ldots, u_{n}(z, t)\right), u_{0}(z)=\left(u_{01}(z), \ldots, u_{0 n}(z)\right) \in \mathbb{R}^{n}$ и аналогично для $u_{1}(z), V(z)$ - матрица взаимодействия (или сила) с элементами $V_{k l}(z), k, l=$ $1, \ldots, n$.

Введем следующие обозначения:

$$
Y(t)=\left(Y^{0}(t), Y^{1}(t)\right) \equiv(u(\cdot, t), \dot{u}(\cdot, t)), \quad Y_{0}=\left(Y_{0}^{0}, Y_{0}^{1}\right) \equiv\left(u_{0}(\cdot), u_{1}(\cdot)\right) .
$$

Тогда задача (1.3) принимает вид

$$
\dot{Y}(t)=\mathcal{A} Y(t), \quad t \in \mathbb{R}, \quad Y(0)=Y_{0} .
$$

Здесь $\mathcal{A}=\left(\begin{array}{cc}0 & 1 \\ -\mathcal{V} & 0\end{array}\right)$, где через $\mathcal{V}$ обозначен оператор свертки с матричным ядром $V$, $\mathcal{V} u=\sum_{z^{\prime} \in \mathbb{Z}^{d}} V\left(z-z^{\prime}\right) u\left(z^{\prime}\right)$. Формально (1.4) - это линейная гамильтонова система с функцией Гамильтона

$$
H(Y)=\frac{1}{2} \sum_{z \in \mathbb{Z}^{d}}\left(\left|u_{1}(z)\right|^{2}+\sum_{z^{\prime} \in \mathbb{Z}^{d}} u_{0}(z) \cdot V\left(z-z^{\prime}\right) u_{0}\left(z^{\prime}\right)\right), \quad Y=\left(u_{0}, u_{1}\right) .
$$

Предполагается, что начальные данные $Y_{0}$ задачи (1.4) принадлежат фазовому пространству $\mathcal{H}_{\alpha}, \alpha \in \mathbb{R}$. По определению фазовое пространство $\mathcal{H}_{\alpha}$ - это гильбертово пространство $\left(\mathbb{R}^{n} \times \mathbb{R}^{n}\right)$-значных функций от $z \in \mathbb{Z}^{d}$ с нормой

$$
\|Y\|_{\alpha}^{2}=\sum_{z \in \mathbb{Z}^{d}}|Y(z)|^{2}\left(1+|z|^{2}\right)^{\alpha}<\infty
$$

На матрицу $V$ налагаются следующие условия.

E1. Существуют константы $C, \gamma>0$ такие, что $\left|V_{k l}(z)\right| \leqslant C e^{-\gamma|z|}$ для всех $z \in \mathbb{Z}^{d}$, $k, l=1, \ldots, n$.

E2. Матрица $V(z)$ действительная и симметричная, т. е. $V_{l k}(-z)=V_{k l}(z) \in \mathbb{R}$ для всех $z \in \mathbb{Z}^{d}, k, l=1, \ldots, n$. 
Обозначим через $\widehat{V}(\theta)$ преобразование Фурье матрицы $V(z)$ :

$$
\widehat{V}(\theta)=\sum_{z \in \mathbb{Z}^{d}} V(z) e^{i z \cdot \theta}, \quad \theta \in \mathbb{T}^{d}
$$

Из условий $\mathbf{E 1}$ и $\mathbf{E 2}$ вытекает, что $\widehat{V}(\theta)$ - действительно-аналитическая, эрмитова, матричнозначная функция от $\theta \in \mathbb{T}^{d}$.

E3. Матрица $\widehat{V}(\theta)$ неотрицательно определена для любых $\theta \in \mathbb{T}^{d}$.

Обозначим через $\Omega(\theta)$ эрмитову неотрицательно-определенную матрицу

$$
\Omega(\theta)=(\widehat{V}(\theta))^{1 / 2} \geqslant 0 .
$$

Через $\omega_{\sigma}(\theta), \sigma=1, \ldots, s$, обозначим собственные значения матрицы $\Omega(\theta)$, которые пронумерованы так, что $0 \leqslant \omega_{1}(\theta)<\omega_{2}(\theta)<\cdots<\omega_{s}(\theta), s \leqslant n$, и через $\Pi_{\sigma}(\theta)-$ соответствующие спектральные проекторы.

Лемма 1 (см. лемму 2.2 в [15]). Пусть выполнены условия E1 и E2. Тогда существует замкнутое множество $\mathcal{C}_{*} \subset \mathbb{T}^{d}$ такое, что:

1) мера Лебега множества $\mathcal{C}_{*}$ равна нулю;

2 ) собственные значения $\omega_{\sigma}(\theta), \sigma=1, \ldots, s$, имеют постоянную кратность на множестве $\mathbb{T}^{d} \backslash \mathcal{C}_{*}$;

3) на множестве $\mathbb{T}^{d} \backslash \mathcal{C}_{*}$ справедливо следующее спектральное разложение: $\Omega(\theta)=\sum_{\sigma=1}^{s} \omega_{\sigma}(\theta) \Pi_{\sigma}(\theta)$, где $\Pi_{\sigma}(\theta)$ - действительно-аналитическая функиия переменной $\theta$.

Обозначим через $\nabla^{2} \omega_{\sigma}(\theta), \theta \in \mathbb{T}^{d} \backslash \mathcal{C}_{*}$, матрицу вторых частных производных функции $\omega_{\sigma}(\theta)$.

E4. Функции $D_{\sigma}(\theta)=\operatorname{det}\left(\nabla^{2} \omega_{\sigma}(\theta)\right), \theta \in \mathbb{T}^{d} \backslash \mathcal{C}_{*}$, не равны тождественно нулю при любом $\sigma=1, \ldots, s$.

Положим

$$
\mathcal{C}_{0}=\left\{\theta \in \mathbb{T}^{d}: \operatorname{det} \widehat{V}(\theta)=0\right\}, \quad \mathcal{C}_{\sigma}=\left\{\theta \in \mathbb{T}^{d} \backslash \mathcal{C}_{*}: D_{\sigma}(\theta)=0\right\} .
$$

Заметим, что лебегова мера множеств $\mathcal{C}_{\sigma}, \sigma=0,1, \ldots, s$, равна нулю [15].

E5. Для любых $\sigma \neq \sigma^{\prime}$ функции $\omega_{\sigma}(\theta) \pm \omega_{\sigma^{\prime}}(\theta)$ при $\theta \in \mathbb{T}^{d} \backslash \mathcal{C}_{*}$ не равны тождественно ненулевым константам.

E6. $\left\|\widehat{V}^{-1}(\theta)\right\| \in L^{1}\left(\mathbb{T}^{d}\right)$.

Очевидно, что если $\mathcal{C}_{0}=\varnothing$, то условие $\mathbf{E} 6$ выполнено.

ЗАмЕчАниЕ 1. Условия E1-E6 выполнены, в частности, в случае кристалла с взаимодействием в соседних точках [15], в котором матрица взаимодействия $V(z)=$ $\left(V_{k l}(z)\right)_{k, l=1}^{n}$ имеет вид

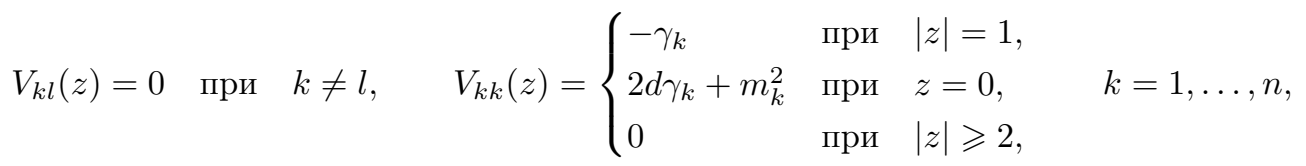

где $\gamma_{k}>0$ и $m_{k} \geqslant 0$. В этом случае уравнение (1.3) принимает вид

$$
\ddot{u}_{k}(z, t)=\left(\gamma_{k} \Delta_{\mathrm{L}}-m_{k}^{2}\right) u_{k}(z, t), \quad k=1, \ldots, n,
$$


где через $\Delta_{\mathrm{L}}$ мы обозначили дискретный оператор Лапласа на решетке $\mathbb{Z}^{d}$,

$$
\Delta_{\mathrm{L}} u(z):=\sum_{e,|e|=1}(u(z+e)-u(z)) .
$$

Следовательно, собственные значения матрицы $\Omega(\theta)$ имеют вид

$$
\omega_{k}(\theta)=\sqrt{2 \gamma_{k}\left(1-\cos \theta_{1}\right)+\cdots+2 \gamma_{k}\left(1-\cos \theta_{d}\right)+m_{k}^{2}}, \quad k=1, \ldots, n .
$$

Очевидно, что условия E1-E5 выполнены, и $\mathcal{C}_{*}=\varnothing$. Если $m_{k}>0$ для всех $k$, то множество $\mathcal{C}_{0}$ пусто, и условие $\mathbf{E 6}$ выполнено. Если $m_{k}=0$ для некоторого $k$, то $\mathcal{C}_{0}=\{0\}$. В этом случае условие $\mathbf{E 6}$ эквивалентно условию $\omega_{k}^{-2}(\theta) \in L^{1}\left(\mathbb{T}^{d}\right)$. Поэтому условия E1-E6 выполнены, если 1) $d \geqslant 3$ или 2) $d=1,2$ и $m_{k}>0$ для Bcex $k$.

ЛЕмма 2 (см. предложение 2.5 в [15]). Пусть выполненъ условия Е1 и Е2 и $\alpha \in \mathbb{R}$. Тогда:

1) для любого $Y_{0} \in \mathcal{H}_{\alpha}$ существует единственное решение $Y(t) \in C\left(\mathbb{R}, \mathcal{H}_{\alpha}\right)$ задачи Коши (1.4);

2) для любого $t \in \mathbb{R}$ оператор $U(t): Y_{0} \mapsto Y(t)$ непрерьвен на $\mathcal{H}_{\alpha}$.

ДокАЗАТЕЛЬСТво этой леммы основано на следующей формуле для решения $Y(t)$ задачи (1.4):

$$
Y(t)=\sum_{z^{\prime} \in \mathbb{Z}^{d}} \mathcal{G}_{t}\left(z-z^{\prime}\right) Y_{0}\left(z^{\prime}\right),
$$

где функция Грина $\mathcal{G}_{t}(z)$ имеет вид

$$
\mathcal{G}_{t}(z):=F_{\theta \rightarrow z}^{-1}\left[e^{\widehat{\mathcal{A}}(\theta) t}\right]=\frac{1}{(2 \pi)^{d}} \int_{\mathbb{T}^{d}} e^{-i z \cdot \theta} e^{\widehat{\mathcal{A}}(\theta) t} d \theta
$$

с матрицей

$$
\widehat{\mathcal{A}}(\theta)=\left(\begin{array}{cc}
0 & 1 \\
-\widehat{V}(\theta) & 0
\end{array}\right), \quad \theta \in \mathbb{T}^{d}
$$

1.2. Семейство начальных мер. Обозначим через $\left\{\mu_{0}^{\varepsilon}, \varepsilon>0\right\}$ семейство начальных мер. Чтобы сформулировать основные условия V1 и V2 для корреляционных функций начальных мер, введем $(2 n \times 2 n)$-матричнозначную функцию $R(r, z)=\left(R^{i j}(r, z)\right)_{i, j=0}^{1}, r \in \mathbb{R}^{d}, z \in \mathbb{Z}^{d}$, удовлетворяющую следующим условиям.

I1. Для любых фиксированных $r \in \mathbb{R}^{d}$ и $i, j=0,1$ справедлива следующая оценка: существуют константы $C>0$ и $\gamma>d$ такие, что

$$
\left|R^{i j}(r, z)\right| \leqslant C(1+|z|)^{-\gamma}, \quad z \in \mathbb{Z}^{d} .
$$

I2. Для любого фиксированного $r \in \mathbb{R}^{d}$ элементы матрицы $\widehat{R}(r, \theta)$ удовлетворяют условиям

$$
\widehat{R}^{00}(r, \theta) \geqslant 0, \quad \widehat{R}^{11}(r, \theta) \geqslant 0, \quad \widehat{R}^{01}(r, \theta)=\widehat{R}^{10}(r, \theta)^{*}, \quad \theta \in \mathbb{T}^{d} .
$$

I3. Для любых $r \in \mathbb{R}^{d}$ и $\theta \in \mathbb{T}^{d}$ матрица $\widehat{R}(r, \theta)$ неотрицательно определена. 
I4. Для любых $\theta \in \mathbb{T}^{d}$ функция $\widehat{R}(\cdot, \theta)$ принадлежит классу $C^{d}$, а функция

$$
r \mapsto \sup _{\theta \in \mathbb{T}^{d}} \max _{i, j=0,1} \max _{\substack{\alpha_{k}=0,1, k=1, \ldots, d}}\left|\frac{\partial^{\alpha_{1}+\cdots+\alpha_{d}}}{\partial r_{1}^{\alpha_{1}} \ldots \partial r_{d}^{\alpha_{d}}} \widehat{R}^{i j}(r, \theta)\right|
$$

равномерно ограничена на ограниченных множествах.

Для вывода уравнения типа Навье-Стокса мы налагаем дополнительное условие.

I4'. Для любого $\theta \in \mathbb{T}^{d}$ функция $\widehat{R}(\cdot, \theta) \in L^{1}\left(\mathbb{R}^{d}\right)$, и существуют константы $C>0$ и $N>d$ такие, что $\sup _{\theta \in \mathbb{T}^{d}}|\widetilde{R}(s, \theta)| \leqslant C(1+|s|)^{-N}$ при $s \in \mathbb{R}^{d}$. Здесь через $\widetilde{R}(s, \theta)$ обозначается преобразование Фурье функции $\widehat{R}(r, \theta)$ по $r$ :

$$
\widetilde{R}(s, \theta)=\int_{\mathbb{R}^{d}} e^{i s \cdot r} \widehat{R}(r, \theta) d r, \quad s \in \mathbb{R}^{d}, \quad \theta \in \mathbb{T}^{d} .
$$

Это условие может быть ослаблено (см. условие $\mathrm{D}^{\prime}$ в [5] для случая $d=1$ ). Можно предположить, что для каждого $\theta \in \mathbb{T}^{d}$ функция $\widehat{R}(\cdot, \theta)$ допускает представление $\widehat{R}(r, \theta)=(2 \pi)^{-d} \int e^{-i s \cdot r} \mu(\theta, d s)$, где $\mu(\theta, d s)$ - некоторая борелевская (комплекснозначная) мера на $\mathbb{R}^{d}$, зависящая от параметра $\theta \in \mathbb{T}^{d}$. Кроме того, существуют константы $C>0$ и $\delta>d$ такие, что для любого $N \in \mathbb{N}$ полная вариация меры $\mu(\theta, \cdot)$ на множестве $|s| \geqslant N$ оценивается следующим образом:

$$
\sup _{\theta \in \mathbb{T}^{d}}|\mu(\theta, \cdot)|_{|s| \geqslant N} \leqslant C(1+N)^{-\delta} .
$$

Обозначим через $\mathbf{E}_{0}^{\varepsilon}$ математическое ожидание по мере $\mu_{0}^{\varepsilon}$ и через

$$
Q_{\varepsilon}^{i j}\left(z, z^{\prime}\right)=\mathbf{E}_{0}^{\varepsilon}\left(Y^{i}(z) \otimes Y^{j}\left(z^{\prime}\right)\right), \quad \varepsilon>0, \quad z, z^{\prime} \in \mathbb{Z}^{d}, \quad i, j=0,1,
$$

- корреляционные функции меры $\mu_{0}^{\varepsilon}$. Предположим, что $\mathbf{E}_{0}^{\varepsilon}(Y(z))=0$, и функции $Q_{\varepsilon}^{i j}\left(z, z^{\prime}\right)$ удовлетворяют следующим условиям.

V1. Для любого $\varepsilon>0$ и для всех $z, z^{\prime} \in \mathbb{Z}^{d}, i, j=0,1$,

$$
\left|Q_{\varepsilon}^{i j}\left(z, z^{\prime}\right)-R^{i j}\left(\varepsilon z, z-z^{\prime}\right)\right| \leqslant \min \left\{C\left(1+\left|z-z^{\prime}\right|\right)^{-\gamma}, \varepsilon b\left|z-z^{\prime}\right|\right\},
$$

где $C, b, \gamma$ - положительные константы, причем $C, \gamma$ взяты из оценки (1.10).

V2. Для любого $\varepsilon>0$ и при всех $z, z^{\prime} \in \mathbb{Z}^{d}, i, j=0,1$ имеет место неравенство $\left|Q_{\varepsilon}^{i j}\left(z, z^{\prime}\right)\right| \leqslant C\left(1+\left|z-z^{\prime}\right|\right)^{-\gamma}$ с теми же константами $C$ и $\gamma$, что в оценке (1.10).

\section{2. ОСНОВНЫЕ РЕЗУЛЬТАТЫ}

Пусть $\mu_{t}^{\varepsilon}$ - вероятностная борелевская мера на $\mathcal{H}_{\alpha}$, которая является распределением случайного решения $Y(t): \mu_{t}^{\varepsilon}(B)=\mu_{0}^{\varepsilon}(U(-t) B)$, где $B \in \mathcal{B}\left(\mathcal{H}_{\alpha}\right)$ и $t \in \mathbb{R}$.

Определим корреляционные функции мер $\mu_{t}^{\varepsilon}$ следующим образом:

$$
Q_{\varepsilon, t}^{i j}\left(z, z^{\prime}\right)=\int\left(Y^{i}(z) \otimes Y^{j}\left(z^{\prime}\right)\right) \mu_{t}^{\varepsilon}(d Y)=\mathbf{E}_{0}^{\varepsilon}\left(Y^{i}(z, t) \otimes Y^{j}\left(z^{\prime}, t\right)\right), \quad i, j=0,1,
$$

где $z, z^{\prime} \in \mathbb{Z}^{d}$, а $Y^{i}(z, t)$ - компоненты случайного решения $Y(t)=\left(Y^{0}(\cdot, t), Y^{1}(\cdot, t)\right)$ задачи (1.4).

Пусть $T_{h}, h \in \mathbb{Z}^{d}$ - группа пространственных сдвигов: $T_{h} Y(x)=Y(x-h), x \in \mathbb{Z}^{d}$. Для всех $\tau \neq 0, r \in \mathbb{R}^{d}$ и $\kappa>0$ определим меры $\mu_{\tau / \varepsilon^{\kappa}, r / \varepsilon}^{\varepsilon}$ следующим образом:

$$
\mu_{\tau / \varepsilon^{\kappa}, r / \varepsilon}^{\varepsilon}(B)=\mu_{\tau / \varepsilon^{\kappa}}^{\varepsilon}\left(T_{[r / \varepsilon]} B\right), \quad B \in \mathcal{B}\left(\mathcal{H}_{\alpha}\right)
$$


ЗАмечАние 2 (см. работу [1]). Пусть $\kappa<1$. Тогда меры $\mu_{\tau / \varepsilon^{\kappa}, r / \varepsilon}^{\varepsilon}$ слабо сходятся к некоторой предельной мере $\mu_{r}$ при $\varepsilon \rightarrow 0$ на пространстве $\mathcal{H}_{\alpha}, \alpha<-d / 2$. По определению это означает, что

$$
\lim _{\varepsilon \rightarrow 0} \int f(Y) \mu_{\tau / \varepsilon^{\kappa}, r / \varepsilon}^{\varepsilon}(d Y)=\int f(Y) \mu_{r}(d Y)
$$

для любого ограниченного непрерывного функционала $f$ на $\mathcal{H}_{\alpha}$. Кроме того доказано, что предельная мера $\mu_{r}$ является гауссовской и не зависит от $\tau$, преобразование Фурье ее корреляционной матрицы имеет вид

$$
\frac{1}{2} \sum_{\sigma=1}^{s} \Pi_{\sigma}(\theta)\left(\widehat{R}(r, \theta)+C_{\sigma}(\theta) \widehat{R}(r, \theta) C_{\sigma}^{*}(\theta)\right) \Pi_{\sigma}(\theta),
$$

где $\Pi_{\sigma}(\theta)$ - спектральный проектор, введенный в лемме 1 , и

$$
C_{\sigma}(\theta)=\left(\begin{array}{cc}
0 & \omega_{\sigma}^{-1}(\theta) \\
-\omega_{\sigma}(\theta) & 0
\end{array}\right), \quad \sigma=1, \ldots, s .
$$

2.1. Уравнение типа Эйлера. Пусть $\kappa=1$, т. е. пространство и время "сжаты" в одном и том же отношении. В этом пункте мы изучим поведение мер $\mu_{\tau / \varepsilon, r / \varepsilon}^{\varepsilon}$ и их корреляционных функций при $\varepsilon \rightarrow 0$.

Введем матрицу $q_{\tau, r}(z)$ при $\tau \neq 0, r \in \mathbb{R}^{d}, z \in \mathbb{Z}^{d}$. Ее преобразование Фурье имеет вид

$$
\hat{q}_{\tau, r}(\theta)=\sum_{\sigma=1}^{s} \Pi_{\sigma}(\theta)\left(\mathbf{M}_{+}^{\sigma}(\tau, r ; \theta)+i \mathbf{M}_{-}^{\sigma}(\tau, r ; \theta)\right) \Pi_{\sigma}(\theta), \quad \theta \in \mathbb{T}^{d} \backslash \mathcal{C}_{*},
$$

где

$$
\begin{aligned}
& \mathbf{M}_{+}^{\sigma}(\tau, r ; \theta)=\frac{R_{+}^{\sigma}(\tau, r ; \theta)+C_{\sigma}(\theta) R_{+}^{\sigma}(\tau, r ; \theta) C_{\sigma}^{*}(\theta)}{2}, \\
& \mathbf{M}_{-}^{\sigma}(\tau, r ; \theta)=\frac{C_{\sigma}(\theta) R_{-}^{\sigma}(\tau, r ; \theta)-R_{-}^{\sigma}(\tau, r ; \theta) C_{\sigma}^{*}(\theta)}{2}
\end{aligned}
$$

и

$$
R_{ \pm}^{\sigma}(\tau, r ; \theta)=\frac{\widehat{R}\left(r+\nabla \omega_{\sigma}(\theta) \tau, \theta\right) \pm \widehat{R}\left(r-\nabla \omega_{\sigma}(\theta) \tau, \theta\right)}{2} .
$$

ТеОрема 1 (см. теорему 4.1 в [1]). Пусть выполнены условия I1-I4, V1, V2 u E1-E6. Тогда для любых $r \in \mathbb{R}^{d}, z, z^{\prime} \in \mathbb{Z}^{d}, \tau \neq 0$ существует предел корреляиионных функиий мер $\mu_{\tau / \varepsilon, r / \varepsilon}^{\varepsilon}$ :

$$
\lim _{\varepsilon \rightarrow 0} Q_{\varepsilon, \tau / \varepsilon}\left([r / \varepsilon]+z,[r / \varepsilon]+z^{\prime}\right)=q_{\tau, r}\left(z-z^{\prime}\right) .
$$

СлЕДСТВИЕ 1. Положим $f_{\sigma}(\tau, r ; \theta)=\Pi_{\sigma}(\theta) \hat{q}_{\tau, r}(\theta) \Pi_{\sigma}(\theta), \sigma=1, \ldots, s . \quad$ Тогдa функиия $f_{\sigma}(\tau, r ; \theta)$ является решением уравнения типа Эйлера:

$$
\begin{aligned}
\partial_{\tau} f_{\sigma}(\tau, r ; \theta) & =i C_{\sigma}(\theta) \nabla \omega_{\sigma}(\theta) \cdot \nabla_{r} f_{\sigma}(\tau, r ; \theta), \quad r \in \mathbb{R}^{d}, \quad \tau>0, \\
f_{\sigma}(0, r ; \theta) & =\frac{1}{2} \Pi_{\sigma}(\theta)\left(\widehat{R}(r, \theta)+C_{\sigma}(\theta) \widehat{R}(r, \theta) C_{\sigma}^{*}(\theta)\right) \Pi_{\sigma}(\theta) .
\end{aligned}
$$


ЗАМЕЧАНИЕ 3. Предположим, что в дополнение к условиям V1 и V2 меры $\mu_{0}^{\varepsilon}$ удовлетворяют условию перемешивания [1]. Тогда для любых $\tau \neq 0$ и $r \in \mathbb{R}^{d}$ меры $\mu_{\tau / \varepsilon, r / \varepsilon}^{\varepsilon}$ слабо сходятся при $\varepsilon \rightarrow 0$ к некоторой гауссовской мере на пространстве $\mathcal{H}_{\alpha}$, $\alpha<-d / 2$ (см. теорему 3.3 в [1]).

ЗАмЕчание 4 . В случае $\kappa \in[1,2)$ корреляционные матрицы мер $\mu_{\tau / \varepsilon^{\kappa}, r / \varepsilon}^{\varepsilon}$ сходятся при $\varepsilon \rightarrow 0$ к тем же матрицам $q_{\tau, r}\left(z-z^{\prime}\right)$, которые введены в теореме 1 . Этот результат может быть доказан аналогично теореме 1.

Результаты теоремы 1 и следствия 1 могут быть сформулированы с использованием матриц Вигнера. Положим $\left(\mathcal{V}^{k} u\right)(x)=F_{\theta \rightarrow x}^{-1}\left[\widehat{V}^{k}(\theta) \hat{u}(\theta)\right], k \in \mathbb{R}$, и

$$
a(x):=\frac{\mathcal{V}^{1 / 4} u_{0}(x)+i \mathcal{V}^{-1 / 4} u_{1}(x)}{\sqrt{2}} \in \mathbb{C}^{n}, \quad x \in \mathbb{Z}^{d} .
$$

Определим матрицу Вигнера (порядка $n \times n)$ следующим образом:

$$
W^{\varepsilon}(\tau, r ; \theta)=\sum_{y \in \mathbb{Z}^{d}} e^{i \theta \cdot y} \mathbf{E}_{\tau / \varepsilon}^{\varepsilon}\left(a^{*}([r / \varepsilon+y / 2]) \otimes a([r / \varepsilon-y / 2])\right), \quad \tau \in \mathbb{R} .
$$

В силу свойств мер $\mu_{0}^{\varepsilon}$ легко доказать, что существует предел

$$
\lim _{\varepsilon \rightarrow 0} W^{\varepsilon}(0, r ; \theta)=W(0, r ; \theta),
$$

где

$$
\begin{aligned}
W(0, r ; \theta)=\frac{1}{2} & \left(\Omega^{1 / 2} \widehat{R}^{00}(r, \theta) \Omega^{1 / 2}+\Omega^{-1 / 2} \widehat{R}^{11}(r, \theta) \Omega^{-1 / 2}+\right. \\
& \left.+i \Omega^{1 / 2} \widehat{R}^{01}(r, \theta) \Omega^{-1 / 2}-i \Omega^{-1 / 2} \widehat{R}^{10}(r, \theta) \Omega^{1 / 2}\right) .
\end{aligned}
$$

Теорема 2 (см. теорему 3.2 в [1]). Пусть выполнены условия V1, V2 и E1-E6. Тогда для любых $r \in \mathbb{R}^{d} u \tau \neq 0$ существует предел (в смысле обобщенных функций)

$$
\lim _{\varepsilon \rightarrow 0} W^{\varepsilon}(\tau, r ; \theta)=W^{\mathrm{p}}(\tau, r ; \theta),
$$

где

$$
W^{\mathrm{p}}(\tau, r ; \theta)=\sum_{\sigma=1}^{s} \Pi_{\sigma}(\theta) W\left(0, r-\tau \nabla \omega_{\sigma}(\theta) ; \theta\right) \Pi_{\sigma}(\theta) .
$$

ЗАмЕчаниЕ 5. Предельные корреляционные матрицы $\hat{q}_{\tau, r}(\theta)=\left(\hat{q}_{\tau, r}^{i j}(\theta)\right)$ (см. равенство (2.2)) выражаются через $W^{\mathrm{p}}(\tau, r ; \theta)$ следующим образом:

$$
\begin{aligned}
\Omega(\theta) \hat{q}_{\tau, r}^{00}(\theta) & =\Omega(\theta)^{-1} \hat{q}_{\tau, r}^{11}(\theta)=\frac{W^{\mathrm{p}}(\tau, r ; \theta)+W^{\mathrm{p}}(\tau, r ;-\theta)^{*}}{2}, \\
\hat{q}_{\tau, r}^{01}(\theta) & =-\hat{q}_{\tau, r}^{10}(\theta)=-\frac{i\left(W^{\mathrm{p}}(\tau, r ; \theta)-W^{\mathrm{p}}(\tau, r ;-\theta)^{*}\right)}{2} .
\end{aligned}
$$

ЗАмЕчАНИЕ 6 . Матрица $f_{\sigma} \equiv \Pi_{\sigma}(\theta) W^{\mathrm{p}}(\tau, r ; \theta) \Pi_{\sigma}(\theta), \sigma=1, \ldots, s$, удовлетворяет уравнению транспорта энергии [1]

$$
\begin{array}{ccc}
\partial_{\tau} f_{\sigma}(\tau, r ; \theta)+\nabla \omega_{\sigma}(\theta) \cdot \nabla_{r} f_{\sigma}(\tau, r ; \theta)=0, & r \in \mathbb{R}^{d}, & \tau>0, \\
f_{\sigma}(0, r ; \theta)=\Pi_{\sigma}(\theta) W(0, r ; \theta) \Pi_{\sigma}(\theta), & r \in \mathbb{R}^{d} . &
\end{array}
$$


2.2. Уравнение типа Навье-Стокса. В этом пункте мы изучим асимптотику при $\varepsilon \rightarrow 0$ корреляционных функций мер $\mu_{\tau / \varepsilon^{\kappa}, r / \varepsilon}^{\varepsilon}$ при $\kappa=2$ и получим следующий член разложения по $\varepsilon$ в уравнении (2.3). Введем матрицу $g_{\varepsilon}(\tau, r ; z), z \in \mathbb{Z}^{d}, r \in \mathbb{R}^{d}$, $\tau \neq 0$, преобразование Фурье которой имеет вид

$$
\hat{g}_{\varepsilon}(\tau, r ; \theta)=\frac{1}{4} \sum_{\sigma=1}^{s} \Pi_{\sigma}(\theta)\left[\sum_{+,-}\left(I \mp i C_{\sigma}(\theta)\right) A_{\varepsilon, \sigma}^{\mp}(\tau, r ; \theta)\left(I \pm i C_{\sigma}^{*}(\theta)\right)\right] \Pi_{\sigma}(\theta),
$$

где $\theta \in \mathbb{T}^{d} \backslash \mathcal{C}_{*}$, матрицы $C_{\sigma}(\theta)$ определены в $(2.1)$,

$$
\begin{gathered}
A_{\varepsilon, \sigma}^{\mp}(\tau, r ; \theta)=\int_{\mathbb{R}^{d}} \widehat{R}\left(r \mp \nabla \omega_{\sigma}(\theta) \tau / \varepsilon-x, \theta\right) K_{\sigma}^{\mp}(\tau, x, \theta) d x, \\
K_{\sigma}^{\mp}(\tau, x, \theta)=F_{y \rightarrow x}^{-1}\left[e^{ \pm i(\tau / 2) y \cdot\left(\nabla^{2} \omega_{\sigma}(\theta)\right) y}\right], \quad x \in \mathbb{R}^{d}
\end{gathered}
$$

(см. также формулу (3.13) ниже).

Теорема 3. Пусть выполнены условия I1-I4', V1, V2 и E1-E6. Тогда для любых $\tau \neq 0, r \in \mathbb{R}^{d} u z, z^{\prime} \in \mathbb{Z}^{d}$ корреляиионные функции мер $\mu_{\tau / \varepsilon^{2}, r / \varepsilon}^{\varepsilon}$ обладают следующей асимптотикой:

$$
\lim _{\varepsilon \rightarrow 0}\left(Q_{\varepsilon, \tau / \varepsilon^{2}}\left([r / \varepsilon]+z,[r / \varepsilon]+z^{\prime}\right)-g_{\varepsilon}\left(\tau, r ; z-z^{\prime}\right)\right)=0,
$$

где матрииа $g_{\varepsilon}(\tau, r ; z)$ определена выше.

Эта теорема доказана в разделе 3 .

Положим $\tau=\varepsilon t$. Заметим, что из формул $(2.5),(2.6)$ следует $\left.\hat{g}_{\varepsilon}(\varepsilon t, r ; \theta)\right|_{\varepsilon=0}=$ $\hat{q}_{t, r}(\theta)$, где $\hat{q}_{t, r}(\theta)$ определена в $(2.2)$. Введем следующие обозначения:

$$
\nabla^{k} \omega_{\sigma}(\theta) \cdot \nabla_{r}^{k} f(r):=\sum_{i_{1}, \ldots, i_{k}=1}^{d} \frac{\partial^{k} \omega_{\sigma}(\theta)}{\partial \theta_{i_{1}} \ldots \partial \theta_{i_{k}}} \frac{\partial^{k} f}{\partial r_{i_{1}} \ldots \partial r_{i_{k}}}, \quad k \in \mathbb{N} .
$$

Следствие 2. Пусть $r \in \mathbb{R}^{d}, t \in \mathbb{R}, \sigma=1, \ldots, s, \theta \in \mathbb{T}^{d}$. Из формулы (2.5) вытекает, что матричнозначная функиия $f_{\sigma} \equiv f_{\sigma}^{\varepsilon}(t, r ; \theta)=\Pi_{\sigma}(\theta) \hat{g}_{\varepsilon}(\varepsilon t, r ; \theta) \Pi_{\sigma}(\theta)$ является решением следующего уравнения:

$$
\partial_{t} f_{\sigma}=i C_{\sigma}(\theta)\left(\nabla \omega_{\sigma}(\theta) \cdot \nabla_{r} f_{\sigma}+\frac{i \varepsilon}{2} \nabla^{2} \omega_{\sigma}(\theta) \cdot \nabla_{r}^{2} f_{\sigma}\right)
$$

с начальным условием (2.4). Это уравнение можно рассматривать как аналог уравнения Навъе-Стокса для нашей модели.

\section{3. ДОКАЗАТЕЛЬСТВО ОСНОВНОГО РЕЗУЛЬТАТА}

Сначала объясним основную идею доказательства. Доказательство опирается на метод "вырезания критических точек" [1], [15], [16] и технику из работы [5], где результат доказан в случае $d=n=1$. Заметим, что в работе [5] налагается более сильное условие на матрицу $V$, чем условия $\mathbf{E 3}, \mathbf{E 4}$. А именно, предполагается, что 
$\omega(\theta)>0$, и множество $\left\{\theta \in[-\pi, \pi]: \omega^{\prime \prime}(\theta)=\omega^{\prime \prime \prime}(\theta)=0\right\}$ пусто. При этих условиях в работе [14] доказана следующая равномерная оценка функции Грина:

$$
\sup _{x \in \mathbb{Z}^{d}}\left|\mathcal{G}_{t}(x)\right| \leqslant C(1+|t|)^{-1 / 3} .
$$

Эта оценка, основанная на методе стационарной фазы, играет важную роль в доказательстве, приведенном в работе [5]. Однако если $n>1$, то функции $\omega_{\sigma}$ могут быть негладкими, а если $d>1$, то множество, на котором $\operatorname{det}\left(\nabla^{2} \omega_{\sigma}(\theta)\right)=0$, может иметь сложную структуру. Поэтому равномерная оценка для $\mathcal{G}_{t}(x)$, подобная $(3.1)$, вообще говоря, невозможна. Чтобы преодолеть эту трудность, введем множество $\mathcal{C} \subset \mathbb{T}^{d}$ лебеговой меры ноль, которое включает все точки, где либо функции $\omega_{\sigma}(\theta)$ негладкие, либо их гессиан равен нулю, и разложим функцию $\mathcal{G}_{t}(x)$ на два слагаемых: $\mathcal{G}_{t}(x)=\mathcal{G}_{t}^{f}(x)+\mathcal{G}_{t}^{g}(x)$, где $\widehat{\mathcal{G}}_{t}^{f}(\theta)$ имеет носитель в окрестности множества $\mathcal{C}$, а $\widehat{\mathcal{G}}_{t}^{g}(\theta)=0-$ в окрестности $\mathcal{C}$. Далее мы докажем, что вклад $\mathcal{G}_{t}^{f}(x)$ незначителен $($ см. $(3.5))$ и $\sup _{x}\left|\mathcal{G}_{t}^{g}(x)\right| \sim(1+|t|)^{-d / 2}$ (см. лемму 4). Эта асимптотика играет важную роль, так как заменяет оценку (3.1), а также упрощает некоторые шаги доказательства из работы [5]. В итоге доказательство сходимости (2.8) сводится к нахождению асимптотики (при $\varepsilon \rightarrow 0$ ) для

$$
Q_{\varepsilon, \tau / \varepsilon^{2}}^{g}\left([r / \varepsilon]+z,[r / \varepsilon]+z^{\prime}\right) \equiv\left(\mathcal{G}_{\tau / \varepsilon^{2}}^{g} * Q_{\varepsilon} * \mathcal{G}_{\tau / \varepsilon^{2}}^{g}\right)\left([r / \varepsilon]+z,[r / \varepsilon]+z^{\prime}\right)
$$

с использованием подхода из работы [5] и методов из работ [1], [15], [16].

3.1. Оценки начальной корреляции. Пусть $p \geqslant 1, n \geqslant 1$. Обозначим через $\ell^{p} \equiv \ell^{p}\left(\mathbb{Z}^{d}\right) \otimes \mathbb{R}^{n}$ пространство последовательностей $f(z)=\left(f_{1}(z), \ldots, f_{n}(z)\right)$ с нормой $\|f\|_{\ell^{p}}=\left(\sum_{z \in \mathbb{Z}^{d}}|f(z)|^{p}\right)^{1 / p}$.

Лемма 3. Пусть выполнено условие V2. Тогда справедливы следующие оценки:

$$
\begin{aligned}
& \sum_{z^{\prime} \in \mathbb{Z}^{d}}\left|Q_{\varepsilon}^{i j}\left(z, z^{\prime}\right)\right| \leqslant C<\infty \quad \text { для всех } \quad z \in \mathbb{Z}^{d}, \\
& \sum_{z \in \mathbb{Z}^{d}}\left|Q_{\varepsilon}^{i j}\left(z, z^{\prime}\right)\right| \leqslant C<\infty \quad \text { для всех } \quad z^{\prime} \in \mathbb{Z}^{d}, \quad i, j=0,1,
\end{aligned}
$$

где константа $C$ не зависит от $z, z^{\prime} \in \mathbb{Z}^{d} u \varepsilon>0$.

СлЕДСТВИЕ 3. Для любых $\Phi, \Psi \in \ell^{2}$

$$
\left|\left\langle Q_{\varepsilon}\left(z, z^{\prime}\right), \Phi(z) \otimes \Psi\left(z^{\prime}\right)\right\rangle\right| \leqslant C\|\Phi\|_{\ell^{2}}\|\Psi\|_{\ell^{2}},
$$

где константа $C$ не зависит от $\varepsilon>0$.

3.2. Метод стационарной фазы. Из формул (1.8) и (1.9) следует, что $\widehat{\mathcal{G}}_{t}(\theta)$ имеет вид

$$
\widehat{\mathcal{G}_{t}}(\theta)=\left(\begin{array}{cc}
\cos (\Omega t) & \sin (\Omega t) \Omega^{-1} \\
-\sin (\Omega t) \Omega & \cos (\Omega t)
\end{array}\right),
$$

где $\Omega=\Omega(\theta)$ - эрмитова матрица, определенная в (1.5). Следовательно, из третьего утверждения леммы 1 , формул

$$
\cos \left(\omega_{\sigma} t\right)=\frac{e^{i \omega_{\sigma} t}+e^{-i \omega_{\sigma} t}}{2}, \quad \sin \left(\omega_{\sigma} t\right)=\frac{e^{i \omega_{\sigma} t}-e^{-i \omega_{\sigma} t}}{2 i}
$$


и равенства $(2.1)$ вытекает, что $\mathcal{G}_{t}(x)$ может быть переписана в виде

$$
\mathcal{G}_{t}(x)=\sum_{\sigma=1}^{s} \sum_{+,-} \int_{\mathbb{T}^{d}} e^{-i x \cdot \theta} e^{ \pm i \omega_{\sigma}(\theta) t} a_{\sigma}^{ \pm}(\theta) d \theta,
$$

где $a_{\sigma}^{ \pm}(\theta)=\Pi_{\sigma}(\theta)\left(I \mp i C_{\sigma}(\theta)\right) / 2$. Для того чтобы применить метод стационарной фазы к интегралу (3.3), требуется гладкость функций $a_{\sigma}^{ \pm}(\theta)$ и $\omega_{\sigma}(\theta)$ по $\theta$. Поэтому выберем некоторые гладкие ветви этих функций и вырежем их сингулярности. Положим

$$
\mathcal{C}=\mathcal{C}_{0} \bigcup \mathcal{C}_{*} \bigcup_{\sigma=1}^{s}\left(\mathcal{C}_{\sigma} \bigcup_{i=1}^{d}\left\{\theta \in \mathbb{T}^{d} \backslash \mathcal{C}_{*}: \frac{\partial^{2} \omega_{\sigma}(\theta)}{\partial \theta_{i}^{2}}=0\right\}\right)
$$

где множество $\mathcal{C}_{*}$ введено в лемме 1, множества $\mathcal{C}_{0}$ и $\mathcal{C}_{\sigma}$ определены в $(1.6)$. Очевидно, что mes $\mathcal{C}=0$ [15]. Зафиксируем $\delta>0$ и выберем разбиение единицы:

$$
f(\theta)+g(\theta)=1, \quad \theta \in \mathbb{T}^{d},
$$

где $f$ и $g$ - неотрицательные функции на $C_{0}^{\infty}\left(\mathbb{T}^{d}\right)$ такие, что

$$
\operatorname{supp} f \subset\left\{\theta \in \mathbb{T}^{d}: \operatorname{dist}(\theta, \mathcal{C})<\delta\right\}, \quad \operatorname{supp} g \subset\left\{\theta \in \mathbb{T}^{d}: \operatorname{dist}(\theta, \mathcal{C}) \geqslant \delta / 2\right\} .
$$

Теперь представим $\mathcal{G}_{t}(x)$ в виде $\mathcal{G}_{t}(x)=\mathcal{G}_{t}^{f}(x)+\mathcal{G}_{t}^{g}(x)$, где

$$
\mathcal{G}_{t}^{f}(x)=F_{\theta \rightarrow x}^{-1}\left[f(\theta) \widehat{\mathcal{G}}_{t}(\theta)\right], \quad \mathcal{G}_{t}^{g}(x)=F_{\theta \rightarrow x}^{-1}\left[g(\theta) \widehat{\mathcal{G}}_{t}(\theta)\right] .
$$

Из равенства Парсеваля, формулы (3.2) и условия Е6 вытекает, что

$$
\begin{aligned}
& \left\|\mathcal{G}_{t}^{f}(\cdot)\right\|_{\ell^{2}}^{2} \leqslant C \int_{\operatorname{dist}(\theta, \mathcal{C})<\delta}\left|\widehat{\mathcal{G}}_{t}(\theta)\right|^{2} d \theta \rightarrow 0 \quad \text { при } \quad \delta \rightarrow 0, \\
& \left\|\mathcal{G}_{t}^{g}(\cdot)\right\|_{\ell^{2}}^{2} \leqslant C \int_{\mathbb{T}^{d}}\left|\widehat{\mathcal{G}}_{t}(\theta)\right|^{2} d \theta \leqslant C_{1}<\infty
\end{aligned}
$$

равномерно по $t \in \mathbb{R}$. Из метода стационарной фазы вытекают следующие оценки для функции $\mathcal{G}_{t}^{g}(x)$.

ЛЕмма 4 (см. лемму 4.2 в [1]). Пусть выполнены условия E1-E4, E6. Тогда:

1) $\sup _{x \in \mathbb{Z}^{d}}\left|\mathcal{G}_{t}^{g}(x)\right| \leqslant C t^{-d / 2}$

2) для любого $p>0$ найдутся $C_{p}, \gamma_{g}>0$ mакие, что $\left|\mathcal{G}_{t}^{g}(x)\right| \leqslant C_{p}(|t|+|x|+1)^{-p}$ при $|x| \geqslant \gamma_{g} t$.

3.3. Доказательство теоремы 3. Доказательство теоремы разобъем на следующие этапы.

ШАГ 1. Из представления (1.7) вытекает, что

$$
Q_{\varepsilon, t}\left(z, z^{\prime}\right)=\mathbf{E}_{0}^{\varepsilon}\left(Y(z, t) \otimes Y\left(z^{\prime}, t\right)\right)=\sum_{x, y \in \mathbb{Z}^{d}} \mathcal{G}_{t}(z-x) Q_{\varepsilon}(x, y) \mathcal{G}_{t}\left(z^{\prime}-y\right)^{\mathrm{T}}
$$

для любых $t \in \mathbb{R}, z, z^{\prime} \in \mathbb{Z}^{d}$. Применим разбиение (3.4), следствие 3 и оценку (3.5) и получим

$$
Q_{\varepsilon, t}\left(z, z^{\prime}\right)=\sum_{x, y \in \mathbb{Z}^{d}} \mathcal{G}_{t}^{g}(z-x) Q_{\varepsilon}(x, y) \mathcal{G}_{t}^{g}\left(z^{\prime}-y\right)^{\mathrm{T}}+o(1)
$$


где $o(1) \rightarrow 0$ при $\delta \rightarrow 0$ равномерно по $t \in \mathbb{R}$ и $z, z^{\prime} \in \mathbb{Z}^{d}$. В частности, полагая $t=\tau / \varepsilon^{2}, z=[r / \varepsilon]+l$ и $z^{\prime}=[r / \varepsilon]+p$, получаем

$$
\begin{aligned}
Q_{\varepsilon, \tau / \varepsilon^{2}} & \equiv Q_{\varepsilon, \tau / \varepsilon^{2}}([r / \varepsilon]+l,[r / \varepsilon]+p)= \\
& =\sum_{x, y \in \mathbb{Z}^{d}} \mathcal{G}_{\tau / \varepsilon^{2}}^{g}([r / \varepsilon]+l-x) Q_{\varepsilon}(x, y) \mathcal{G}_{\tau / \varepsilon^{2}}^{g}([r / \varepsilon]+p-y)^{\mathrm{T}}+o(1)= \\
& =\sum_{x, y \in \mathbb{Z}^{d}} \mathcal{G}_{\tau / \varepsilon^{2}}^{g}(l+x) Q_{\varepsilon}([r / \varepsilon]-x,[r / \varepsilon]-y) \mathcal{G}_{\tau / \varepsilon^{2}}^{g}(p+y)^{\mathrm{T}}+o(1) .
\end{aligned}
$$

Положим $c=\gamma_{g}+\max \{|l|,|p|\}$. Тогда из второго утверждения леммы 4 и условия V2 вытекает, что суммирование в ряде (3.6) можно проводить только по области $x, y \in\left[-c \tau / \varepsilon^{2}, c \tau / \varepsilon^{2}\right]^{d}$.

ШАГ 2. Асимптотика функции $Q_{\varepsilon, \tau / \varepsilon^{2}}$ не изменится, если в правой части (3.6) заменить $Q_{\varepsilon}([r / \varepsilon]-x,[r / \varepsilon]-y)$ на $R(\varepsilon[r / \varepsilon]-\varepsilon x, y-x)$. Действительно, во-первых, из первого утверждения леммы 4 и условия V1 следует, что

$$
\begin{gathered}
\mid \sum_{x, y \in\left[-c \tau / \varepsilon^{2}, c \tau / \varepsilon^{2}\right]^{d}} \mathcal{G}_{\tau / \varepsilon^{2}}^{g}(l+x)\left(Q_{\varepsilon}([r / \varepsilon]-x,[r / \varepsilon]-y)-R(\varepsilon[r / \varepsilon]-\varepsilon x, y-x)\right) \times \\
\times \mathcal{G}_{\tau / \varepsilon^{2}}^{g}(p+y)^{\mathrm{T}} \mid \leqslant C \sum_{y \in \mathbb{Z}^{d}} \min \left\{(1+|x-y|)^{-\gamma}, \varepsilon b|x-y|\right\} .
\end{gathered}
$$

Во-вторых,

$$
\sum_{y \in \mathbb{Z}^{d}} \min \left\{(1+|x-y|)^{-\gamma}, \varepsilon b|x-y|\right\} \sim C \varepsilon^{(\gamma-d) /(\gamma+1)} \rightarrow 0, \quad \varepsilon \rightarrow 0,
$$

так как $\gamma>d$.

Используя лемму 4 и свойства функции $R$, заменим область суммирования в рядах на $x, y \in \mathbb{Z}^{d}$. Следовательно,

$$
Q_{\varepsilon, \tau / \varepsilon^{2}}=\sum_{x \in \mathbb{Z}^{d}} \mathcal{G}_{\tau / \varepsilon^{2}}^{g}(l+x) \sum_{y \in \mathbb{Z}^{d}} R(\varepsilon[r / \varepsilon]-\varepsilon x, y-x) \mathcal{G}_{\tau / \varepsilon^{2}}^{g}(p+y)^{\mathrm{T}}+o(1), \quad \varepsilon \rightarrow 0 .
$$

ШАГ 3. Применяя равенство Парсеваля, перепишем $Q_{\varepsilon, \tau / \varepsilon^{2}}$ в виде

$$
\begin{aligned}
Q_{\varepsilon, \tau / \varepsilon^{2}}= & \frac{1}{(2 \pi)^{2 d}} \sum_{x \in \mathbb{Z}^{d}} \int_{\mathbb{T}^{2 d}} e^{-i l \cdot \theta^{\prime}+i p \cdot \theta+i x \cdot\left(\theta-\theta^{\prime}\right)} \widehat{\mathcal{G}}_{\tau / \varepsilon^{2}}^{g}\left(\theta^{\prime}\right) \widehat{R}(\varepsilon[r / \varepsilon]-\varepsilon x, \theta) \times \\
& \times \widehat{\mathcal{G}}_{\tau / \varepsilon^{2}}^{g}(\theta)^{*} d \theta d \theta^{\prime}+o(1) .
\end{aligned}
$$

Сделаем замену переменных в последнем интеграле, $\left(\theta, \theta^{\prime}\right) \rightarrow(\theta, \varphi), \varphi=\theta-\theta^{\prime}$, и перепишем $Q_{\varepsilon, \tau / \varepsilon^{2}}$ в виде

$$
Q_{\varepsilon, \tau / \varepsilon^{2}}=\frac{1}{(2 \pi)^{d}} \int_{\mathbb{T}^{d}} e^{-i(l-p) \cdot \theta} I_{\varepsilon}(\theta) \widehat{\mathcal{G}}_{\tau / \varepsilon^{2}}^{g}(\theta)^{*} d \theta+o(1)
$$

где $I_{\varepsilon}(\theta)$ обозначает матричнозначную функцию

$$
I_{\varepsilon}(\theta)=\sum_{x \in \mathbb{Z}^{d}} \frac{1}{(2 \pi)^{d}} \int_{\mathbb{T}^{d}} e^{i(l+x) \cdot \varphi} \widehat{\mathcal{G}}_{\tau / \varepsilon^{2}}^{g}(\theta-\varphi) \widehat{R}(\varepsilon[r / \varepsilon]-\varepsilon x, \theta) d \varphi .
$$


Применим формулу суммирования Пуассона (см., например, [20]) и получим

$$
\sum_{x \in \mathbb{Z}^{d}} e^{i x \cdot \varphi} \widehat{R}(\varepsilon[r / \varepsilon]-\varepsilon x, \theta)=\varepsilon^{-d} e^{i[r / \varepsilon] \cdot \varphi} \sum_{n \in \mathbb{Z}^{d}} \widetilde{R}(-\varphi / \varepsilon-2 \pi n / \varepsilon, \theta),
$$

где $\widetilde{R}(\cdot, \theta)$ обозначает преобразование Фурье функции $\widehat{R}(\cdot, \theta)$ (см. (1.11)). Подставим последнее выражение в (3.8) и получим

$$
I_{\varepsilon}(\theta)=\frac{1}{(2 \pi \varepsilon)^{d}} \int_{[-\pi, \pi]^{d}} e^{i(l+[r / \varepsilon]) \cdot \varphi} \widehat{\mathcal{G}}_{\tau / \varepsilon^{2}}^{g}(\theta-\varphi)\left(\sum_{n \in \mathbb{Z}^{d}} \widetilde{R}(-\varphi / \varepsilon-2 \pi n / \varepsilon, \theta)\right) d \varphi .
$$

ШАГ 4. Из условия $\mathbf{I} \mathbf{4}^{\prime}$ следует, что

$$
\varepsilon^{-d} \sum_{n \neq 0} \widetilde{R}(-\varphi / \varepsilon-2 \pi n / \varepsilon, \theta) \rightarrow 0 \quad \text { при } \quad \varepsilon \rightarrow 0
$$

равномерно по $\theta \in \mathbb{T}^{d}, \varphi \in[-\pi, \pi]^{d}$. Таким образом, из (3.9) и (3.10) вытекает, что

$$
I_{\varepsilon}(\theta)=\frac{1}{(2 \pi \varepsilon)^{d}} \int_{[-\pi, \pi]^{d}} e^{i \varphi \cdot(l+[r / \varepsilon])} \widehat{\mathcal{G}}_{\tau / \varepsilon^{2}}^{g}(\theta-\varphi) \widetilde{R}(-\varphi / \varepsilon, \theta) d \varphi+o(1) .
$$

Сделаем замену переменных $\varphi \rightarrow-\varepsilon \varphi$ в последнем интеграле, получим

$$
I_{\varepsilon}(\theta)=\frac{1}{(2 \pi)^{d}} \int_{[-\pi / \varepsilon, \pi / \varepsilon]^{d}} e^{-i \varepsilon \varphi \cdot(l+[r / \varepsilon])} \widehat{\mathcal{G}}_{\tau / \varepsilon^{2}}^{g}(\theta+\varepsilon \varphi) \widetilde{R}(\varphi, \theta) d \varphi+o(1) .
$$

Применим представление (3.3) для $\widehat{\mathcal{G}}_{t}^{g}(\theta)$ :

$$
\widehat{\mathcal{G}}_{t}^{g}(\theta)=\sum_{\sigma=1}^{s} \sum_{+,-} e^{ \pm i \omega_{\sigma}(\theta) t} h_{\sigma}^{\mp}(\theta), \quad h_{\sigma}^{\mp}(\theta)=g(\theta) \Pi_{\sigma}(\theta) \frac{I \mp i C_{\sigma}(\theta)}{2} .
$$

Используя это соотношение, перепишем $I_{\varepsilon}(\theta)$ в виде

$$
\begin{gathered}
I_{\varepsilon}(\theta)=\frac{1}{(2 \pi)^{d}} \sum_{\sigma=1}^{s} \sum_{+,-} \int_{[-\pi / \varepsilon, \pi / \varepsilon]^{d}} e^{-i \varepsilon \varphi \cdot(l+[r / \varepsilon])} e^{ \pm i \omega_{\sigma}(\theta+\varepsilon \varphi) \tau / \varepsilon^{2}} h_{\sigma}^{\mp}(\theta+\varepsilon \varphi) \widetilde{R}(\varphi, \theta) d \varphi+ \\
+o(1)=\frac{1}{(2 \pi)^{d}} \sum_{\sigma=1}^{s} \sum_{+,-} h_{\sigma}^{\mp}(\theta) \int_{\mathbb{R}^{d}} e^{-i \varphi \cdot r} e^{ \pm i \omega_{\sigma}(\theta+\varepsilon \varphi) \tau / \varepsilon^{2}} \widetilde{R}(\varphi, \theta) d \varphi+o(1)
\end{gathered}
$$

при $\varepsilon \rightarrow 0$, так как $e^{-i \varepsilon \varphi \cdot l}-1=O(\varepsilon)$ и $e^{-i \varphi \cdot \varepsilon[r / \varepsilon]}-e^{-i \varphi \cdot r}=O(\varepsilon)$.

ШАГ 5. Применим разложение Тейлора для $\omega_{\sigma}(\theta+\varepsilon \varphi)$ :

$$
\omega_{\sigma}(\theta+\varepsilon \varphi) \frac{\tau}{\varepsilon^{2}}=\omega_{\sigma}(\theta) \frac{\tau}{\varepsilon^{2}}+\varphi \cdot \nabla \omega_{\sigma}(\theta) \frac{\tau}{\varepsilon}+\frac{\tau}{2} \varphi \cdot H_{\sigma}(\theta) \varphi+O(\varepsilon),
$$

где $H_{\sigma}(\theta)$ обозначает матрицу $\nabla^{2} \omega_{\sigma}(\theta)$. Следовательно,

$$
I_{\varepsilon}(\theta)=\sum_{\sigma=1+,-}^{s} h_{\sigma}^{\mp}(\theta) e^{ \pm i \omega_{\sigma}(\theta) \tau / \varepsilon^{2}} A_{\varepsilon, \sigma}^{\mp}(\tau, r ; \theta)+o(1), \quad \varepsilon \rightarrow 0,
$$


где (см. формулы $(2.6)$ и (2.7))

$$
\begin{aligned}
A_{\varepsilon, \sigma}^{\mp}(\tau, r ; \theta) & =\frac{1}{(2 \pi)^{d}} \int_{\mathbb{R}^{d}} e^{-i \varphi \cdot\left(r \mp \nabla \omega_{\sigma}(\theta) \tau / \varepsilon\right)} e^{ \pm i(\tau / 2) \varphi \cdot H_{\sigma}(\theta) \varphi} \widetilde{R}(\varphi, \theta) d \varphi= \\
& =\int_{\mathbb{R}^{d}} \widehat{R}\left(r \mp \nabla \omega_{\sigma}(\theta) \tau / \varepsilon-x, \theta\right) K_{\sigma}^{\mp}(\tau, x, \theta) d x .
\end{aligned}
$$

Функция $K_{\sigma}^{\mp}(\tau, x, \theta), \tau>0, x \in \mathbb{R}^{d}, \theta \in \mathbb{T}^{d} \backslash \mathcal{C}$, имеет вид

$$
K_{\sigma}^{\mp}(\tau, x, \theta)=F_{\varphi \rightarrow x}^{-1}\left[e^{ \pm i(\tau / 2) \varphi \cdot H_{\sigma}(\theta) \varphi}\right]=\frac{e^{ \pm i \pi s / 4}}{(2 \pi \tau)^{d / 2}} \frac{e^{\mp i x \cdot H_{\sigma}^{-1}(\theta) x /(2 \tau)}}{\sqrt{\left|D_{\sigma}(\theta)\right|}},
$$

где $D_{\sigma}(\theta)=\operatorname{det} H_{\sigma}(\theta)$ и $s-$ сигнатура матрицы $H_{\sigma}(\theta)$.

Наконец, подставим $(3.12)$ в $(3.7)$, применим разложение $(3.11)$ к $\widehat{\mathcal{G}}_{\tau / \varepsilon^{2}}^{g}(\theta)^{*}$ и получим

$$
\begin{aligned}
Q_{\varepsilon, \tau / \varepsilon^{2}}= & \frac{1}{(2 \pi)^{d}} \int_{\mathbb{T}^{d}} e^{-i(l-p) \cdot \theta}\left(\sum_{\sigma=1}^{s} \sum_{+,-} h_{\sigma}^{\mp}(\theta) e^{ \pm i \omega_{\sigma}(\theta) \tau / \varepsilon^{2}} A_{\varepsilon, \sigma}^{\mp}(\tau, r ; \theta)\right) \times \\
& \times\left(\sum_{\sigma^{\prime}=1}^{s} \sum_{+,-} e^{ \pm i \omega_{\sigma^{\prime}}(\theta) \tau / \varepsilon^{2}} h_{\sigma^{\prime}}^{\mp}(\theta)^{*}\right) d \theta+o(1), \quad \varepsilon \rightarrow 0 .
\end{aligned}
$$

Следовательно, чтобы найти асимптотику $Q_{\varepsilon, \tau / \varepsilon^{2}}$, достаточно изучить поведение при $\varepsilon \rightarrow 0$ следующих интегралов, входящих в $Q_{\varepsilon, \tau / \varepsilon^{2}}$ :

$$
I_{\sigma \sigma^{\prime}}^{ \pm}(\varepsilon) \equiv \frac{1}{(2 \pi)^{d}} \int_{\mathbb{T}^{d}} e^{-i(l-p) \cdot \theta} e^{i\left(\omega_{\sigma}(\theta) \pm \omega_{\sigma^{\prime}}(\theta)\right) \tau / \varepsilon^{2}} h_{\sigma}^{-}(\theta) A_{\varepsilon, \sigma}^{-}(\tau, r ; \theta) h_{\sigma^{\prime}}^{\mp}(\theta)^{*} d \theta,
$$

$\sigma, \sigma^{\prime}=1, \ldots, s$. Заметим, что функция $h_{\sigma}^{-}(\theta) A_{\varepsilon, \sigma}^{-}(\tau, r ; \theta) h_{\sigma^{\prime}}^{\mp}(\theta)^{*} \in L^{1}\left(\mathbb{T}^{d}\right)$, что вытекает из условий $\mathbf{E} 6$ и $\mathbf{I} 4^{\prime}$. Кроме того, тождества $\omega_{\sigma}(\theta) \pm \omega_{\sigma^{\prime}}(\theta) \equiv$ const $_{ \pm}$в экспоненте в случае const \pm $\neq 0$ невозможны в силу условия E5. Осциллирующие интегралы с $\omega_{\sigma}(\theta) \pm \omega_{\sigma^{\prime}}(\theta) \not \equiv$ const $_{ \pm}$стремятся к нулю при $\varepsilon \rightarrow 0$ в силу теоремы Лебега-Римана. Следовательно, только интегралы с $\omega_{\sigma}(\theta)-\omega_{\sigma^{\prime}}(\theta) \equiv 0$ вносят вклад, так как если $\omega_{\sigma}(\theta)+\omega_{\sigma^{\prime}}(\theta) \equiv 0$, то $\omega_{\sigma}(\theta) \equiv \omega_{\sigma^{\prime}}(\theta) \equiv 0$, что невозможно в силу условия $\mathbf{E 4}$. Таким образом, если $\sigma \neq \sigma^{\prime}$, то $I_{\sigma \sigma^{\prime}}^{ \pm}(\varepsilon)=o(1)$ при $\varepsilon \rightarrow 0$. Если $\sigma=\sigma^{\prime}$, то $I_{\sigma \sigma}^{+}(\varepsilon)=o(1)$ и

$$
I_{\sigma \sigma}^{-}(\varepsilon)=\frac{1}{(2 \pi)^{d}} \int_{\mathbb{T}^{d}} e^{-i(l-p) \cdot \theta} h_{\sigma}^{-}(\theta) A_{\varepsilon, \sigma}^{-}(\tau, r ; \theta) h_{\sigma}^{+}(\theta)^{*} d \theta+o(1), \quad \varepsilon \rightarrow 0 .
$$

Это завершает доказательство асимптотики (2.8).

\section{Поправки высшего порядка}

ПРИЛОЖЕНИЕ

Чтобы получить дополнительные члены порядка $\varepsilon^{2}, \varepsilon^{3}$ и т. д. в уравнении $(2.10)$, необходимо изучить асимптотику при $\varepsilon \rightarrow 0$ корреляционных функций мер $\mu_{\tau / \varepsilon^{\kappa}, r / \varepsilon}^{\varepsilon}$ при $\kappa>2$.

TеОрема 4. Пусть $\kappa \geqslant 2, r \in \mathbb{R}^{d}, \tau>0$. Тогда для всех $z, z^{\prime} \in \mathbb{Z}^{d}$

$$
Q_{\varepsilon, \tau / \varepsilon^{\kappa}}\left([r / \varepsilon]+z,[r / \varepsilon]+z^{\prime}\right)-g_{\varepsilon}^{[\kappa]}\left(\tau, r ; z-z^{\prime}\right) \rightarrow 0, \quad \varepsilon \rightarrow 0 .
$$


Преобразование Фуръе матрицы $g_{\varepsilon}^{k}(\tau, r ; z), k=2,3, \ldots$, имеет вид

$$
\hat{g}_{\varepsilon}^{k}(\tau, r ; \theta)=\frac{1}{4} \sum_{\sigma=1}^{s} \Pi_{\sigma}(\theta)\left(I \mp i C_{\sigma}(\theta)\right) A_{\varepsilon, \sigma}^{\mp, k}(\tau, r ; \theta)\left(I \pm i C_{\sigma}^{*}(\theta)\right) \Pi_{\sigma}(\theta),
$$

где

$$
\begin{gathered}
A_{\varepsilon, \sigma}^{\mp, k}(\tau, r ; \theta)=\int_{\mathbb{R}^{d}} \widehat{R}\left(r \mp \nabla \omega_{\sigma}(\theta) \tau / \varepsilon^{k-1}-x, \theta\right) K_{\varepsilon, \sigma}^{\mp, k}(\tau, x, \theta) d x, \quad \theta \in \mathbb{T}^{d} \backslash \mathcal{C}, \\
K_{\varepsilon, \sigma}^{\mp, k}(\tau, x, \theta)=F_{y \rightarrow x}^{-1}\left[\exp \left\{ \pm i \frac{\tau}{\varepsilon^{k-2}}\left(\frac{\nabla^{2} \omega_{\sigma}(\theta) \cdot y^{2}}{2 !}+\cdots+\frac{\nabla^{k} \omega_{\sigma}(\theta) \cdot y^{k}}{k !}\right)\right\}\right]
\end{gathered}
$$

и по определению

$$
\nabla^{k} \omega_{\sigma}(\theta) \cdot y^{k}:=\sum_{i_{1}, \ldots, i_{k}=1}^{d} \frac{\partial^{k} \omega_{\sigma}(\theta)}{\partial \theta_{i_{1}} \ldots \partial \theta_{i_{k}}} y_{i_{1}} \ldots y_{i_{k}}, \quad y=\left(y_{1}, \ldots, y_{d}\right) \in \mathbb{R}^{d}
$$

Заметим, что при $k=2$ матрица $\hat{g}_{\varepsilon}^{k}(\tau, r ; \theta)$ совпадает с матрицей $\hat{g}_{\varepsilon}(\tau, r ; \theta)$ из формулы (2.5). Доказательство теоремы 4 аналогично доказательству теоремы 3.

Положим $\tau=\varepsilon^{k-1} t, k \geqslant 2$. Тогда матрица $\Pi_{\sigma}(\theta) \hat{g}_{\varepsilon}^{k}\left(\varepsilon^{k-1} t, r ; \theta\right) \Pi_{\sigma}(\theta)$ (обозначим ее через $\left.f_{\sigma}^{\varepsilon}(t, r ; \theta)\right)$ является решением следующего уравнения:

$$
\partial_{t} f_{\sigma}^{\varepsilon}(t, r ; \theta)=i C_{\sigma}(\theta) P_{\varepsilon}^{k}\left(\theta, \partial_{r}\right) f_{\sigma}^{\varepsilon}(t, r ; \theta), \quad t>0, \quad r \in \mathbb{R}^{d},
$$

с начальным условием (2.4). Здесь $P_{\varepsilon}^{k}\left(\theta, \partial_{r}\right):=\sum_{p=1}^{k} \frac{(i \varepsilon)^{p-1}}{p !} \nabla^{p} \omega_{\sigma}(\theta) \cdot \nabla_{r}^{p}$ (см. обозначение $(2.9))$. Обозначим через $f_{\sigma}^{i j}, i, j=0,1$, элементы матрицы $f_{\sigma}^{\varepsilon}(t, r ; \theta)$. Тогда $f_{\sigma}^{11}=\omega_{\sigma}^{2}(\theta) f_{\sigma}^{00}, f_{\sigma}^{10}=-f_{\sigma}^{01}$, и уравнения (П.1) могут быть переписаны в виде

$$
\partial_{t} f_{\sigma}^{00}=-\frac{i}{\omega_{\sigma}(\theta)} P_{\varepsilon}^{k}\left(\theta, \partial_{r}\right) f_{\sigma}^{01}, \quad \partial_{t} f_{\sigma}^{01}=i \omega_{\sigma}(\theta) P_{\varepsilon}^{k}\left(\theta, \partial_{r}\right) f_{\sigma}^{00}
$$

Благодарности. Работа частично поддержана РФФИ (грант № 09-01-00288).

\section{Список литературы}

[1] T. V. Dudnikova, H. Spohn, Markov Process. Relat. Fields, 12:4 (2006), 645-578, arXiv: math-ph/0505031.

[2] T. V. Dudnikova, J. Math. Phys., 51:8 (2010), 083301, 25 pp., arXiv: 0905.4806.

[3] C. Boldrighini, R. L. Dobrushin, Yu. M. Sukhov, J. Statist. Phys., 31:3 (1983), 577-616.

[4] R. L. Dobrushin, A. Pellegrinotti, Yu. M. Suhov, L. Triolo, J. Statist. Phys., 43:3-4 (1986), 571-607.

[5] R. L. Dobrushin, A. Pellegrinotti, Yu. M. Suhov, L. Triolo, J. Statist. Phys., 52:1-2 (1988), 423-439.

[6] R. L. Dobrushin, A. Pellegrinotti, Yu. M. Suhov, J. Statist. Phys., 61:1-2 (1990), 387-402.

[7] S. Olla, R. S. R. Varadhan, H.-T. Yau, Commun. Math. Phys., 155:3 (1993), 523-560.

[8] R. Esposito, R. Marra, H.-T. Yau, Commun. Math. Phys., 182:2 (1996), 395-455.

[9] J. Quastel, H.-T. Yau, Ann. Math., 148:1 (1998), 51-108.

[10] A. De Masi, N. Ianiro, A. Pellegrinotti, E. Presutti, "A survey of the hydrodynamical behavior of many-particle systems", Nonequilibrium Phenomena II: From Stochastics to Hydrodynamics, Stud. Statist. Mech., XI, eds. J. L. Lebowitz, E. W. Montroll, North-Holland Phys. Publ., Amsderdam, 1984, 123-294. 
[11] Р. Л. Добрушин, Я. Г. Синай, Ю. М. Сухов, “Динамические системы статистической механики", Динамические системы - 2, Итоги науки и техн. Сер. Соврем. пробл. мат. Фундам. направления, 2, ВИНИТИ, М., 1985, 235-284.

[12] J. Fritz, An Introduction to the Theory of Hydrodynamic Limits, Lect. Math. Sci., 18, The Graduate School of Mathematics, Univ. Tokyo, Tokyo, 2001.

[13] H. Spohn, Large Scale Dynamics of Interacting Particles, Texts Monogr. Phys., XI, Springer, Berlin, 1991.

[14] C. Boldrighini, A. Pellegrinotti, L. Triolo, J. Statist. Phys., 30:1 (1983), 123-155.

[15] T. V. Dudnikova, A. I. Komech, H. Spohn, J. Math. Phys., 44:6 (2003), 2596-2620, arXiv: math-ph/0210039.

[16] T. V. Dudnikova, A. I. Komech, N. Mauser, J. Statist. Phys., 114:3-4 (2004), 1035-1083, arXiv: math-ph/0211017.

[17] T. V. Dudnikova, A. I. Komech, H. Spohn, Markov Process. Relat. Fields, 8:1 (2002), 43-80, arXiv: math-ph/0508044.

[18] Т. В. Дудникова, А. И. Комеч, Теория вероятн. и ее примен., 50:4 (2005), 675-710.

[19] T. V. Dudnikova, A. I. Komech, Russ. J. Math. Phys., 12:3 (2005), 301-325, arXiv: math-ph/0508053.

[20] Э. Ч. Титчмарш, Введение в теорию интегралов Фуръе, Гостехиздат, М., Л., 1948.

Поступила в редакцию 19.01.2011, после доработки 26.02.2011 